\title{
COMPORTAMIENTO DE HÍBRIDOS DE MAÍZ (Zea mays L.) EN SISTEMA TRANSITORIO CON CACAO (Theobroma cacao L.) EN LA PARROQUIA LODANA, ECUADOR
}

\author{
AUTORES: Julio Gabriel Ortega ${ }^{1}$ \\ Jhon Jairo Figueroa Venegas ${ }^{2}$ \\ Ricardo Limongi Andrade 3 \\ Raquel Vera Velázquez ${ }^{4}$ \\ Blanca Indacochea Ganchozo 5
}

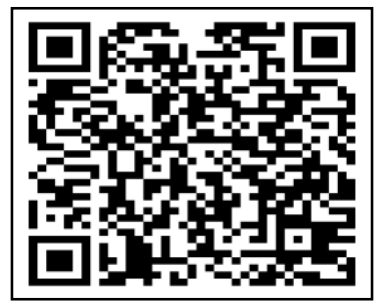

DIRECCIÓN PARA CORRESPONDENCIA: julio.gabriel@unesum.edu.ec

Fecha de recepción: 10/11/2021

Fecha de aceptación: 02/01/2022

\section{RESUMEN}

La investigación tuvo como objetivo estudiar el efecto del maíz (Zea mays) como sistema asociado con el cultivo de cacao (Theobroma cacao) en la parroquia Lodana del cantón Santa Ana. Se utilizó el diseño experimental de Bloques Completos al Azar (DBCA) con tres repeticiones en arreglo factorial 3 x 8, donde los factores fueron (A) los híbridos de maíz (INIAP H-601, 602 y 603), (B) los clones de cacao [EET-800, 801, 450, 454, 575, 576, 103, CCN51(testigo)]. Las variables de repuesta evaluadas fueron: floración masculina y femenina, acame de raíz y tallo, enfermedades foliares, altura de planta e inserción de mazorca, número/plantas/parcela, longitud y diámetro de mazorca, \% mazorcas podridas, número/hilera de grano por mazorca, peso de 1000 granos, rendimiento y prolificidad. Para cacao las variables de respuesta fueron: Altura de planta a $20 \mathrm{~cm}$, diámetro de tallo y cobertura vegetal, la cual es obtenida mediante "CANOPEO" una herramienta que permite seguir el crecimiento de los cultivos mediante el cálculo de la fracción de cubierta vegetal. Los resultados mostraron que la mayor producción y productividad de maíz asociado con cacao fue el híbrido INIAP H-601 con rendimiento de $1.59 \mathrm{t} / \mathrm{ha}$, y prolificidad de 1.51 en un promedio de dos mazorcas por cada planta. Además, presentó el menor porcentaje de mazorcas podridas. La influencia del maíz sobre el crecimiento del cacao fue positiva lo que se evidenció con la altura de planta siendo el clon de

\footnotetext{
1 Docente investigador, Universidad Estatal del Sur de Manabí (UNESUM), Jipijapa, Ecuador. E-mail: julio.gabriel@unesum.edu.ec. ORCID: https://orcid.org/0000-0001-9776-9235.

${ }^{2}$ Ingeniero graduado, Carrera Agropecuaria, Facultad de Ciencias Naturales y de la Agricultura, Universidad Estatal del Sur de Manabí. Jipijapa. Manabí.

${ }^{3}$ Investigador. INIAP, Portoviejo. Ecuador. E-mail: jricardo.limongi@ gmail.com . ORCID: https://orcid.org/00000001-9478-5301.

${ }^{4}$ Docente investigadora, Universidad Estatal del Sur de Manabí (UNESUM), Jipijapa, Ecuador. . E-mail: veraraquel@unesum.edu.ec. ORCID: https://orcid.org/0000-0002-5071-7523

5 Docente investigadora Universidad Estatal del Sur de Manabí. Jipijapa. Manabí. Ecuador. E-mail: blanca.indacochea@unesum.edu.ec . ORCID: https://orcid.org/0000-0003-4741-2435.
} 
Julio Gabriel, Jhon Figueroa, Ricardo Limongi, Raquel Vera, Blanca Indacochea

cacao EET-800 el que alcanzó mayor altura con $37.37 \mathrm{~cm}$, mientras el más bajo fue para el clon CCN-51(testigo) con $22.73 \mathrm{~cm}$. La cobertura de copa tuvo un crecimiento positivo para el clon de cacao EET-801 con un promedio de $36.51 \%$.

PALABRAS CLAVE: Prolificidad, cultivos asociados; producción; productividad; clones.

\section{BEHAVIOR OF CORN (Zea mays L.) HYBRIDS IN TRANSITORY SYSTEM WITH CACAO (Theobroma cacao L.) IN LODANA PARISH, ECUADOR}

\section{ABSTRACT}

The objective of the research was to study the effect of corn (Zea mays) as a system associated with the cultivation of cocoa (Theobroma cacao) in the Lodana parish of the Santa Ana canton. The experimental design of Complete Random Blocks (DBCA) with three repetitions in a 3 x 8 factorial arrangement, where the factors were (A) the corn hybrids (INIAP H-601, 602 and 603), (B) the cocoa clones [EET-800, 801, 450, 454, 575, 576, 103, CCN-51 (control)]. The response variables evaluated were: male and female flowering, root and stem lodging, foliar diseases, plant height and ear insertion, number / plants / plot, ear length and diameter, \% rotten ears, number / row of grain per ear, weight of 1000 grains, yield and prolificacy. For cocoa, the response variables were: Plant height at $20 \mathrm{~cm}$, stem diameter and plant cover, which is obtained through "CANOPEO" a tool that allows to follow the growth of crops by calculating the plant cover fraction. The results showed that the highest production and productivity of maize associated with cocoa was the INIAP H-601 hybrid with a yield of $1.59 \mathrm{t} / \mathrm{ha}$. And prolificacy of 1.51 in an average of two ears per plant. In addition, it had the lowest percentage of rotten ears. The influence of corn on cacao growth was positive, which was evidenced by plant height, with the EET-800 cacao clone the one that reached the highest height with $37.37 \mathrm{~cm}$, while the lowest was for the CCN-51 clone (control) with $22.73 \mathrm{~cm}$. The crown cover had a positive growth for the cocoa clone EET-801 with an average of $36.51 \%$.

KEYWORDS: Prolificacy; associated crops; production; productivity; clones.

\section{INTRODUCCIÓN}

El "asocio de cultivos" es uno de los principios básicos de la "agricultura ecológica", toda vez que permiten hacer un uso más eficiente y racional de los recursos disponibles, generar sistemas productivos menos dependientes de factores externos y preservar el medio ambiente. Mediante la integración del conocimiento tradicional adaptado a las condiciones locales y culturales, con el conocimiento técnico-científico se puede llegar a una agricultura moderna, sostenible y competitiva (Rojas, 2015).

En el litoral ecuatoriano, el cultivo de maíz es de suma importancia por ser la región con mayor producción de maíz amarillo duro (Zea mays L.) "La superficie sembrada de maíz amarillo duro a nivel nacional durante el año 2016 fue de 246.367 ha (SINAGAP, 2016). A nivel nacional el maíz duro seco está localizado principalmente en la Región Costa. Las provincias de Los Ríos, Manabí y Guayas sumaron el $80.20 \%$ de la superficie total cosechada de este producto. Se observa que la provincia de los Ríos es la que más se dedica a este cultivo, con una participación del $40.01 \%$ a nivel nacional, de igual forma su producción es la más alta, concentrando el $40.07 \%$ 
de las toneladas métricas del grano, mientras Manabí y Guayas concentran el $29.41 \%$ y $12.33 \%$ de la producción nacional respectivamente. (Salazar et al., 2016).

En Manabí, Ecuador, el cultivo de maíz cobra importancia económica, ya que pequeños y medianos productores se dedican a su siembra durante la época lluviosa, bajo condiciones de laderas, con eliminación de rastrojos que son amontonados y quemados para el establecimiento y manejo del cultivo en suelo "limpio" y la reducción de problemas de insectos plagas. (Limongi, 2011). El cacao es el producto ecuatoriano de exportación tradicional con mayor historia en la economía del país, involucra alrededor de 100.000 familias de productores. El cacao fino o de aroma es uno de los más cotizados en el mercado internacional por sus características particulares de aroma y sabor. El cacao históricamente ha estado asociado a la vida republicana del país. Aún más cuando los pilares de nuestra economía se levantaron gracias a la producción y comercialización de la "pepa de oro" Asociación Nacional de exportadores de Cacao (Anecacao, 2017).

Según Anecacao (2017), Ecuador fue el mayor exportador mundial de cacao durante el período 1880 - 1915. No obstante, se perdió este estatus debido al ataque de dos enfermedades conocidas como la Moniliasis y la Escoba de Bruja. Entre 1915 y 1930 la producción disminuyó en un 63\% (de 40.000 toneladas métricas a 15.000 toneladas métricas). El cacao es un cultivo tradicional en Manabí y su producción ha sido durante años la fuente de ingresos para muchas familias manabitas. Según Instituto de investigaciones agropecuaria (Iniap, 2017) Manabí, existen 100.961 ha sembradas, de éstas 52.546 son en monocultivos y 48.415 asociados. Debido que el cultivo de cacao se enmarca en un sistema agroforestal, principalmente plátano, frutales y maderables, los cuales al mismo tiempo que le proveen sombra al cacao, le permiten al agricultor tener otras alternativas de ingresos. Los sistemas de este tipo se caracterizan por conservar el suelo y el ambiente, en la medida en que son grandes generadores de biomasa; además de los beneficios que proporciona en la parte ambiental, el cacao es un cultivo tradicional de economías campesinas (Iniap, 2017).

Por otro lado, el cacao es una especie sensible a la intensidad lumínica, situación que puede provocar defoliación de las puntas de las ramas, y aumentar la acción de insectos chupadores que intensifican su actividad debido a un inadecuando sombramiento (Quiroz, 2010). Así mismo por sus características genéticas requiere de ciertos niveles de sombra para su normal desarrollo (Enríquez, 2010). Generalmente todas estas actividades de siembra o establecimiento se realizan mediante diversos sistemas de cultivos mixtos, en donde cobran importancia aquellos destinados a la seguridad alimentaria como los de cultivo ciclo corto, por ejemplo, maíz, maní, arroz, frejol, caupí entre otros. Principalmente porque reducen los costos de establecimiento, se optimiza el uso eficiente de la tierra (Enríquez, 2010).

En muchas regiones de América Latina y el Caribe, el cultivo de cacao es bajo sombra, en sistemas complejos, multiestratos que incluyen especies de usos múltiples (Somarriba y Beer, 1994), tendencia que es encontrada a nivel del Litoral ecuatoriano, principalmente en la provincia de Manabí donde el $48.42 \%$ de sus plantaciones se encuentran en asociación con otras especies (Melo y Hollander, 2013). Debido a que la producción cacaotera del Ecuador está estrechamente relacionada a las condiciones del ecosistema, determina un rendimiento diferente al de otros países productores. En general se consideran factores importantes que influyen en el rendimiento: 
Julio Gabriel, Jhon Figueroa, Ricardo Limongi, Raquel Vera, Blanca Indacochea

la imperfecta distribución de las lluvias, escasez de horas luz, la presencia de enfermedades como la monilia y escoba de bruja, edad avanzada de los árboles, pérdida de fertilidad del suelo, falta de zonificación del cultivo, problemas de comercialización interna y la escasa respuesta técnica a estos problemas suscitados. Sin embargo, no existen datos que determinen la eficiencia de incorporar cultivos anuales como el maíz en el cacao durante el establecimiento del cultivo de cacao.

La investigación se realizó con el fin de dar a conocer aquellas experiencia obtenidas en el establecimiento cacao-maíz en la cual algunas de las ventajas comparativas que ofrece este sistema se refiere al aprovechamiento en el uso de la tierra y la pronta generación de ingresos, que lo convierten en una alternativa para los pequeños y medianos productores debido a que la aplicación y el manejo de estos componentes favorecen al cacao, proveyéndolo de ciertos niveles de sombra, que permitirán mantener un balance de las condiciones climáticas, al optimizar el uso de radiación solar, agua y nutrientes; así como el manejo de plagas y la polinización. Por tal motivo este trabajo de investigación pretende generar información relevante del cultivo de maíz y su efecto en el cultivo de cacao, lo cual contribuiría nuevas orientaciones para futuros trabajos alrededor de los sistemas asociados.

En la actualidad el rendimiento de los cultivos se ha visto afectado por el manejo de la tierra en formas inadecuadas, dando lugar a la existencia de insectos-plagas, que ocasionan bajos rendimientos de estos productos, afectando la economía de los agricultores. Además, la falta de información relevante para los agricultores, determina la importancia de los cultivos transitorios alrededor del cultivo de cacao; así como la ausencia de inventarios que determinan cual es la contribución económica en la rentabilidad y productividad del cultivo. El interés por este tipo de sistemas se debe a la necesidad de encontrar opciones para la baja producción y degradación de la tierra, manteniendo en todo momento el principio de desarrollo sostenible. Estos sistemas pueden contribuir a solucionar problemas en el uso de los recursos naturales debido a las funciones biológicas y socioeconómicas que cumplen (Alviar, 2016). Por lo cual, en busca de alternativas para mejorar la producción agrícola, especialmente el cultivo de maíz cuando se presenta en un sistema de producción transitorio se investigará e identificará los niveles de producción y rendimiento bajo el sistema asociado en el cultivo de cacao.

Por todo lo antes expuesto la investigación tiene el objetivo de estudiar el efecto del maíz (Zea mays) como sistema asociado con el cultivo de cacao (Theobroma cacao) en la parroquia Lodana del cantón Santa Ana.

\section{DESARROLLO}

Este estudio se realizó en la parroquia Lodana, Cantón Santa Ana, Manabí, sus características climáticas son: altitud de $44 \mathrm{msnm}$, humedad relativa promedio de $82 \%$, precipitación medio anual de $550 \mathrm{~mm}$, suelo tipo vertic ustropets, temperatura promedio de $24.6^{\circ} \mathrm{C}$ y categoría de bosque seco pre montano subtropical, se encuentra ubicada en el Km 20 vía Santa Ana, cantón Portoviejo, Manabí.

Ubicación geográfica del sitio experimental (Lodana-Manabí): longitud $80^{\circ} 23^{\prime} \mathrm{W}$ y latitud $01^{\circ} 12^{\prime}$ S Altitud 44. Características climáticas y edáficas del sito experimental (Lodana-Manabí): temperatura promedio $\left({ }^{\circ} \mathrm{C}\right) 24.6$, precipitación anual $(\mathrm{mm}) 550$, heliofanía media anual (horas) 1266, topografía plana, textura franco arcilloso $\mathrm{Ph}$ 6.5-7

138 UNESUM-Ciencias. Publicación cuatrimestral. Vol. 6, Año 2022, No. 2 (Especial Agropecuaria) 
Publicación cuatrimestral. Vol. 6, No. 2 (Especial Agropecuaria), Año 2022. Pág. 135-152 COMPORTAMIENTO DE HIBRIDOS DE MAIZ (Zea Mays L.) EN SISTEMA TRANSITORIO CON CACAO

Los factores en estudios son dos: Factor A: Híbridos de maíz (H); H1= INIAP H-601; H2= INIAP H-602; H3= INIAP H-603

Factor B: Clones de cacao (C): C1: EET - 800; C2: EET - 801; C3: EET - 450; C4: EET - 454; C5: EET - 575; C6: EET - 576; C7: EET - 103; C8: CCN - 51 (Testigo)

Tabla 1. Tratamientos estuvieron dados por la combinación de dos factores en estudio, donde se utilizó el CCN-51 como testigo.

\begin{tabular}{|c|c|c|c|}
\hline $\mathbf{N}^{\mathbf{0}}$ & NOMENCLATURA & Híbridos de maíz & Clones de cacao \\
\hline 1. & $\mathrm{H} 1 \mathrm{XC1}$ & INIAP H-601 & EET-800 \\
\hline 2. & $2 \mathrm{H} 1 \mathrm{XC} 2$ & INIAP H-601 & EET-801 \\
\hline 3. & $\mathrm{H} 1 \mathrm{XC} 3$ & INIAP H-601 & EET-450 \\
\hline 4. & $\mathrm{H} 1 \mathrm{XC} 4$ & INIAP H-601 & EET-454 \\
\hline 5. & $\mathrm{H} 1 \mathrm{XC} 5$ & INIAP H-601 & EET-575 \\
\hline 6. & H1XC6 & INIAP H-601 & EET-576 \\
\hline 7. & $\mathrm{H} 1 \mathrm{XC} 7$ & INIAP H-601 & EETP-103 \\
\hline 8. & $\mathrm{H} 1 \mathrm{XC} 8$ & INIAP H-601 & $\mathrm{CCN}-51$ \\
\hline 9. & $\mathrm{H} 2 \mathrm{XC} 1$ & INIAP H-602 & EET-800 \\
\hline 10. & $\mathrm{H} 2 \mathrm{XC} 2$ & INIAP H-602 & EET-801 \\
\hline 11. & $\mathrm{H} 2 \mathrm{XC} 3$ & INIAP H-602 & EET-450 \\
\hline 12. & $\mathrm{H} 2 \mathrm{XC} 4$ & INIAP H-602 & EET-454 \\
\hline 13. & $\mathrm{H} 2 \mathrm{XC} 5$ & INIAP H-602 & EET-575 \\
\hline 14. & $\mathrm{H} 2 \mathrm{XC} 6$ & INIAP H-602 & EET-576 \\
\hline 15. & $\mathrm{H} 2 \mathrm{XC} 7$ & INIAP H-602 & EET-103 \\
\hline 16. & $\mathrm{H} 2 \mathrm{XC} 8$ & INIAP H-602 & $\mathrm{CCN}-51$ \\
\hline 17. & $\mathrm{H} 3 \mathrm{XC} 1$ & INIAP H-603 & EET-800 \\
\hline 18. & $\mathrm{H} 3 \mathrm{XC} 2$ & INIAP H-603 & EET-801 \\
\hline 19. & $\mathrm{H} 3 \mathrm{XC} 3$ & INIAP H-603 & EET-450 \\
\hline 20. & $\mathrm{H} 3 \mathrm{XC} 4$ & INIAP H-603 & EET-454 \\
\hline 21. & $\mathrm{H} 3 \mathrm{XC} 5$ & INIAP H-603 & EET-575 \\
\hline 22. & H3XC6 & INIAP H-603 & EET-576 \\
\hline \multirow[t]{2}{*}{23.} & $\mathrm{H} 3 \mathrm{XC} 7$ & INIAP H-603 & EET-103 \\
\hline & $\mathrm{H} 3 \mathrm{XC} 8$ & INIAP H-603 & CCN-51 \\
\hline
\end{tabular}

Fuente: Figueroa y Gabriel (2019)

Se utilizó un diseño de Bloques Completamente al azar (DBCA) con tres repeticiones en arreglo factorial 3x8 ( 3 híbridos de maíz * 8 clones de cacao). 
Julio Gabriel, Jhon Figueroa, Ricardo Limongi, Raquel Vera, Blanca Indacochea

Dando a lugar a 72 unidades experimentales. La situación más práctica es aquella donde se forman los elementos muéstrales sobre cada unidad experimental (Gabriel et al., 2017)

\section{Características del experimento}

Tabla 2. Delineamiento Experimental

\begin{tabular}{lc}
\hline Hileras útiles & 3 \\
Número de plantas por unidad experimenta & 40 \\
Número de plantas por parcela útil & 18 \\
Número de plantas evaluadas en parcela útil & 10 \\
Distancia entre hileras & $3 \mathrm{~m}$ \\
Distancia entre plantas & $0,20 \mathrm{~m}$ \\
Distancia entre repeticiones & $8 \mathrm{~m}$ \\
Longitud de parcela & $15 \mathrm{~m}$ \\
Ancho de parcela & $44 \mathrm{~m}$ \\
Área Total de la Parcela & $660 \mathrm{~m}^{2}$ \\
Área útil de la parcela & $24 \mathrm{~m}^{2}$ \\
Área útil del ensayo & $576 \mathrm{~m}^{2}$ \\
Área total del ensayo & $9324 \mathrm{~m}^{2}$ \\
\hline
\end{tabular}

Fuente: Figueroa y Gabriel (2019)

\section{Análisis estadístico}

Previo al análisis de varianza se realizó la prueba de distribución normal y de homogeneidad de varianzas de las variables de respuestas evaluadas (Gabriel et al., 2017). Sobre la base del módulo definido se realizaron análisis de varianzas (tabla 4) para probar la hipótesis acerca de los efectos fijos, así como comparaciones de medias de los tratamientos mediante la prueba de Tukey al 5\% de probabilidad. El análisis de varianza también sirvió para estimar los componentes de varianza de los efectos aleatorios, los análisis fueron realizados utilizando el paquete estadístico INFOSTAT (Gabriel et al., 2017).

Los bloques se definen como un conjunto de unidades experimentales homogéneas dentro de sí y heterogéneas entre sí. En cada bloque están representados todos los tratamientos.

\section{Variables de respuesta}

Para la floración Masculina, se registró el número de días transcurridos desde la siembra hasta que el $50 \%$ de plantas presentaron las espiga o panoja (Centro Internacional del Maíz y el Trigo (CIMMYT, 1995).

Para la floración femenina, se registró el número de días transcurrido desde la siembra hasta que el $50 \%$ de plantas presentaron los estigmas expuestos, con al menos $2 \mathrm{~cm}$ de largo (CIMMYT, 1995).

Las enfermedades foliares se registraron alrededor de los 80 a 90 días después de la siembra. La calificación se realizó de acuerdo a la escala de 1 a 5 propuesta por el (CIMMYT, 1995), donde:

140 UNESUM-Ciencias. Publicación cuatrimestral. Vol. 6, Año 2022, No. 2 (Especial Agropecuaria) 
1=infección débil, 2=infección ligera, 3=infección moderada, 4=infección severa y 5= infección muy severa (CIMMYT, 1995).

Se evaluó una semana antes de la cosecha y se expresó en porcentajes, respecto al total de planta (CIMMYT, 1995).

Acame de tallo. Se refiere al total de plantas en la parcela neta que presentaron el tallo quebrado bajo la mazorca superior. Se evaluó una semana antes de la cosecha y se expresó en porcentaje, respecto al total de plantas (CIMMYT, 1995).

Altura de planta $(\mathrm{cm})$. Se evaluaron 10 plantas, desde la base de la planta hasta donde la espiga o panoja empieza a ramificarse. Se registró el valor promedio después de la floración expresada en centímetro (CIMMYT, 1995).

Altura de inserción mazorca $(\mathrm{cm})$. Se evaluaron 10 plantas, desde la base de la planta hasta el nudo de inserción de la mazorca superior. Se registró el valor promedio después de la floración expresada en centímetro (CIMMYT, 1995).

Longitud de mazorca $(\mathrm{cm})$. Se evaluó desde la base en su inserción con el pedúnculo hasta su ápice después de la cosecha, el valor registrado correspondió a el promedio de 10 mazorcas expresado en centímetros (CIMMYT, 1995).

Diámetro de mazorca $(\mathrm{cm})$. Se evaluó con un calibrador en la parte central de 10 mazorcas elegidas al azar después de la cosecha y el valor promedio se expresó en centímetros (CIMMYT, 1995).

Porcentajes de mazorca podridas. Se determinó contando el número de mazorcas que presentaron pudrición con respecto a total de mazorcas cosechadas dentro de la parcela útil y se expresó en porcentaje (Gabriel et al., 2017).

Número de hileras por mazorca. Se contó el número de hileras de mazorca por las parcelas netas para luego realizar el valor promedio.

Peso de 1000 granos (g). Esta variable se determinó después del desgrane de 10 mazorcas y se registró el peso de 100 granos tomados al alzar y se expresado en gramos (CIMMYT, 1995).

Rendimiento t/ha. Se calculó esta variable, ajustando al 13\% de humedad, utilizando la siguiente fórmula (IBPGR, 1991).

Rendimiento $\mathrm{t} / \mathrm{h}_{a}=\frac{(P C x D x M S) X 1000}{87 x A P}$

Donde: $\mathrm{PC}=$ Peso de campo en $(\mathrm{kg})$, total de mazorcas cosechadas por parcela. $\mathrm{D}=$ Proporción de grano, expresado en decimales.

Proporción de grano $=\frac{\text { Peso de grano }(5 \text { mazorcas })}{\text { Peso total }(5 \text { mazorcas })}$

MS = Materia seca (100 $-\%$ de humedad), expresado en forma decimal. $87=$ Factor para ajustar el grano al $13 \%$ de humedad. AP = Área de la parcela, expresada en metros cuadrados.

Prolificidad. Mediante esta variable se verifico el número de mazorcas obtenida por cada planta. Variables de respuesta en clones de cacao 
Julio Gabriel, Jhon Figueroa, Ricardo Limongi, Raquel Vera, Blanca Indacochea

Altura de la planta. Se determinó el crecimiento de las plantas de cada uno de los individuos a evaluar ( $\mathrm{n}=9$ ), en cada unidad experimental, se midió desde el nivel del suelo hasta ápice de la planta utilizando primera evaluación a los 56 días de establecida la plantación de cacao, y posteriormente a los 105 días, hasta los 146 días de la plantación.

Diámetro de la planta. Se determinó el diámetro de tallo a $20 \mathrm{~cm}$ del suelo, con la ayuda de un calibrador, a cada uno de los individuos a evaluar $(n=9)$, en cada unidad experimental, se realizó la primera evaluación a los 56 días de establecida la plantación de cacao, y posteriormente a los 105 días, hasta los 146 días de la plantación.

Cobertura vegetal. Se evaluó la cobertura vegetal con la ayuda del programa canopeo, a cada uno de los individuos a evaluar $(n=9)$, en cada unidad experimental, donde se realizaron tres evaluaciones.

Durante el desarrollo de la investigación se efectuaron las siguientes labores en el cultivo de maíz:

Semilla. Previo a la siembra se realizó el tratamiento de la semilla con el insecticida Thiodicard (15 cc/kg de semilla) tratando que la semilla quede impregnada en su totalidad.

Siembra. La siembra del ensayo experimental fue realizada el 6 de septiembre en EE-T Portoviejo, se realizó de forma manual, colocando una semilla por cada sitio cada $20 \mathrm{~cm}$, en surco de $7.4 \mathrm{~cm}$ de longitud.

Control de maleza. El control de maleza se ejecutó con controles manuales o mecánicos (moto guaraña), luego se inició con aplicaciones químicas, utilizando el herbicida gramoxone en dosis de $200 \mathrm{cc} / 201$ de agua, estas labores se realizaron de la misma forma y frecuencia para todo el experimento.

Riego. El riego se lo realizo por surcos, de acuerdo a los tratamientos en estudios llenando cada surco en su totalidad. Cabe indicar que, por el establecimiento del experimento a inicios de la época seca, se dará riegos uniformes de tal manera que el cultivo se establezca.

Fertilización. Se procedió a aplicar nitrógeno (urea), a los 15, 30 y 45 días antes de la floración, ubicando al costado de la planta siempre y cuando exista humedad.

Control de plagas y enfermedades. Se realizaron aplicaciones necesarias para reducir el problema de insectos plagas y enfermedades en las diferentes etapas del cultivo.

Cosecha. La cosecha se realizó de forma manual a los 120 días después de la siembra, una vez que el cultivo alcanzo su madurez fisiológica.

\section{Resultados}

\section{Análisis de normalidad.}

Para ilustrar el análisis, solamente se considerará un ejemplo de los tratamientos. El análisis mostró que el sesgo (Skewness) de la curva es igual $\mathrm{A}=0$, esto indica que la curva no presenta ningún sesgo. Por lo tanto, la Kurtosis es platicurtica, obteniendo un coeficiente de variación (C.V \%) de $15.23 \%$. Que está indicando que está dentro de los rangos permitidos para este tipo de investigación. Esto denota que los datos tienen un ajuste normal y no necesitan transformación.

142 UNESUM-Ciencias. Publicación cuatrimestral. Vol. 6, Año 2022, No. 2 (Especial Agropecuaria) 
En el análisis de homogeneidad de varianzas mediante la prueba Chi-Cuadrada, mostró que las medias de varianza son homogéneas $(\mathrm{ChiSq}<0.0001)$, por lo que ambos análisis sugieren proceder con los análisis de varianza y comparación de medias.

Floración masculina. En el análisis de varianza para esta variable (Tabla 3), reportó diferencias estadísticas altamente significativas al $\mathrm{P}<0.01$ de probabilidad entre híbridos de maíz. El coeficiente de variación es de 1.89 y el promedio general de la floración fue de 56 días.

Floración femenina. El análisis de varianza para esta variable (Tabla 3), se reportó diferencia estadística altamente significativas al $\mathrm{P}<0.01$ de probabilidad entre híbridos de maíz. El coeficiente de variación fue de $1.66 \%$ y el promedio general fue 58 días.

Acame de raíz. La (Tabla 3), muestra que el análisis de varianza para esta variable, reportó diferencias altamente significativas al $\mathrm{P}<0.01$ de probabilidad entre híbridos de maíz. El coeficiente es de variación fue $42.88 \%$.

Acame de tallo y enfermedades foliares. Al realizar el análisis de varianza para estas variables (tabla 3) no hubo diferencias significativas entre los híbridos en estudio. Los coeficientes de variación fueron de 34.29 y $25.23 \%$ respectivamente.

Tabla 3. Cuadrados medios para floración masculina, femenina, acame de tallo, acame de raíz y enfermedades foliares, en un sistema de cultivo asociado cacao-maíz en (Lodana Manabí)

\begin{tabular}{|c|c|c|c|c|c|c|}
\hline \multirow{3}{*}{$\begin{array}{l}\text { Fuentes de } \\
\text { variación }\end{array}$} & \multirow{3}{*}{$\begin{array}{l}\text { Grados de } \\
\text { libertad }\end{array}$} & \multicolumn{5}{|c|}{ Cuadrados medios } \\
\hline & & \multicolumn{2}{|c|}{ Floración } & \multicolumn{2}{|l|}{ Acame } & \multirow{2}{*}{$\begin{array}{l}\text { Enfermedades } \\
\text { foliares }\end{array}$} \\
\hline & & $\begin{array}{l}\text { Masculina } \\
\text { (días) }\end{array}$ & $\begin{array}{l}\text { Femenina } \\
\text { (días) }\end{array}$ & $\begin{array}{l}\text { Tallo } \\
(\%)\end{array}$ & Raíz (\%) & \\
\hline Maíz & 2 & $66.33 * *$ & $82.29 * *$ & $0.08 \mathrm{~ns}$ & $3.06 * *$ & $0.17 \mathrm{~ns}$ \\
\hline Cacao & 7 & $0.91 \mathrm{~ns}$ & $0.41 \mathrm{~ns}$ & $0.07 \mathrm{~ns}$ & $0.34 \mathrm{~ns}$ & $0.06 \mathrm{~ns}$ \\
\hline Maíz*Cacao & 14 & $0.84 \mathrm{~ns}$ & $0.47 \mathrm{~ns}$ & $0.09 \mathrm{~ns}$ & $0.76 \mathrm{~ns}$ & $0.17 \mathrm{~ns}$ \\
\hline Error & 48 & 1.12 & 0.94 & 0.16 & 0.59 & 0.15 \\
\hline Total & 71 & & & & & \\
\hline CV (\%) & & 1.89 & 1.66 & 34.29 & 42.88 & 25.23 \\
\hline
\end{tabular}

**: Altamente Significativo al $1 \%$ de probabilidad *: Significativo al 5\% de probabilidad. ns: Diferencias estadísticas no significativas, CV: Coeficiente de variación

En la (Tabla 4), se presentan los valores promedios y la prueba de Tukey al $5 \%$ efectuada para floración masculina, femenina, acame de tallo, acame de raíz y enfermedades foliares. En los tres híbridos de maíz se observó que la floración masculina presenta tres rangos de significación estadística, siendo el híbrido INIAP H-603 el más tardío con 57 días; y el híbrido INIAP-601 el más precoz con 54 días a la floración.

La floración femenina presenta tres rangos de significación estadística (Tabla 4), siendo el híbrido INIAP H-603 el más tardío con 60 días; mientras que el híbrido INIAP-601 es el más precoz con 56 días promedio a floración. 
Julio Gabriel, Jhon Figueroa, Ricardo Limongi, Raquel Vera, Blanca Indacochea

En acame de raíz se observaron dos rangos de significación estadística, el mayor correspondió a los híbridos de maíz INIAP H-602 y 603 con 2.04 y $1.98 \%$ respectivamente; y el rango más bajo se presentó en el híbrido de maíz INIAP H-601 con 1.4\% promedio.

No hubo diferencia significativa para ninguna de las variables de respuestas en el cultivo de cacao.

Tabla 4. Valores promedios para floración masculina, femenina, acame de tallo, acame de raíz y enfermedades foliares, en 3 híbridos de maíz evaluados durante la época seca del año 2017 en cultivo asociado cacao-maíz en Lodana-Manabí (INIAP Portoviejo).

\begin{tabular}{|c|c|c|c|c|c|}
\hline \multirow[t]{2}{*}{ Tratamientos } & \multirow{2}{*}{\begin{tabular}{|l|} 
Floración \\
$\begin{array}{l}\text { Masculina } \\
\text { (días) }\end{array}$
\end{tabular}} & \multicolumn{3}{|c|}{ Acame } & $\begin{array}{l}\text { Enfermedades } \\
\text { foliares }\end{array}$ \\
\hline & & $\begin{array}{l}\text { Femenina } \\
\text { (días) }\end{array}$ & Raíz (\%) & Tallo (\%) & \\
\hline
\end{tabular}

Factor A:

Híbridos de maíz

$\begin{array}{lllllllll}\text { INIAP H-601 } & 54.06 & \mathrm{c} & 55.36 & \mathrm{c} & 1.4 & \mathrm{a} & 1.16 \mathrm{a} & 2 \mathrm{a} \\ \text { INIAP H-602 } & 56.18 & \mathrm{~b} & 58.76 & \mathrm{~b} & 2.04 & \mathrm{~b} & 1.20 \mathrm{a} & 2 \mathrm{a} \\ \text { INIAP H-603 } & 57.35 & \mathrm{a} & 60.02 & \mathrm{a} & 1.98 & \mathrm{~b} & 1.08 \mathrm{a} & 1 \mathrm{a}\end{array}$

Medias con la misma letra no muestras diferencias significativas al $\mathrm{P}<0.05$ de probabilidad.

Altura de planta. En el análisis de varianza para altura de planta (tabla 5), se detectaron diferencias estadísticas significativas al $5 \%$ de probabilidad entre los híbridos de maíz. Se observa un coeficiente de variación de $8.61 \%$ y una altura de $192 \mathrm{~cm}$.

Altura de inserción mazorca. Al realizar el análisis de varianza para esta variable (tabla 5), se detectaron diferencias significativas al $\mathrm{P}<0.05$ de probabilidad entre híbridos de maíz. Se obtuvo una altura de inserción de $92 \mathrm{~cm}$ y un coeficiente de variación de $12.44 \%$.

$\mathbf{N}^{\circ}$ de planta/parcela. En esta variable, el análisis de varianza (tabla 5), reportó diferencias altamente significativas al $\mathrm{P}<0.01$ de probabilidad entre híbridos. Se observa un coeficiente de variación de $26.79 \%$ y un promedio de 59 planta por parcela.

Tabla 5. Cuadrados medios para cuatro variables agronómicas registradas en 3 híbridos de maíz evaluadas durante la época seca 2017. En un sistema de cultivo asociado cacao-maíz en LodanaManabí.

\begin{tabular}{|c|c|c|c|c|}
\hline \multirow{3}{*}{$\begin{array}{l}\text { Fuentes } \\
\text { variación }\end{array}$} & \multirow{3}{*}{$\begin{array}{ll}\text { Grados de } \\
\text { libertad }\end{array}$} & \multicolumn{2}{|c|}{ Cuadrados medios } & \multirow[b]{3}{*}{$\mathbf{N}^{\circ}$ de planta } \\
\hline & & \multicolumn{2}{|l|}{ Altura } & \\
\hline & & Planta $(\mathrm{cm})$ & inser/Mazorca $(\mathrm{cm})$ & \\
\hline Maíz & 2 & $9367.33 *$ & $4390.74 *$ & $3326.77 * *$ \\
\hline Cacao & 7 & $300.32 \mathrm{~ns}$ & $115.08 \mathrm{~ns}$ & $38.82 \mathrm{~ns}$ \\
\hline Maíz*cacao & 14 & $530.99 \mathrm{~ns}$ & $191.6 \mathrm{~ns}$ & $278.28 \mathrm{~ns}$ \\
\hline Error & 48 & 273.34 & 130.16 & 244.2 \\
\hline Total & 71 & & & \\
\hline
\end{tabular}

144 UNESUM-Ciencias. Publicación cuatrimestral. Vol. 6, Año 2022, No. 2 (Especial Agropecuaria) 
Publicación cuatrimestral. Vol. 6, No. 2 (Especial Agropecuaria), Año 2022. Pág. 135-152 COMPORTAMIENTO DE HIBRIDOS DE MAIZ (Zea Mays L.) EN SISTEMA TRANSITORIO CON CACAO

CV (\%)

8.61

12.44

26.79

**,*: Significativo al 1 y 5\% de probabilidad. Ns: Diferencias estadísticas no significativas.

La tabla 6 , muestra los valores promedios y la prueba Tukey al $\mathrm{P}<0.05$ de probabilidad; aquí se observa que la variable altura de planta en los híbridos de maíz presenta dos rangos de significancia estadística, el mayor corresponde al maíz híbrido INIAP H-601 e INIAP H-602 con 202.21 y $204.50 \mathrm{~cm}$ en promedio y el rango más bajo se presenta en el híbrido INIAP H603 con promedio de $168.46 \mathrm{~cm}$.

La inserción de mazorca presenta dos rangos de significancia estadística, el mayor corresponde a los híbridos de maíz INIAP H-601 e INIAP H-602 con 95.92 y 102.65 cm, respectivamente y el rango más bajo corresponde al INIAP H-603 con $76.02 \mathrm{~cm}$.

El número de plantas por parcela en los híbridos de maíz evaluados presenta dos rangos de significancia estadísticas, el mayor corresponde a los híbridos de maíz INIAP H-601 e INIAP H602 con 66.71 y 64.44, mientras el más bajo presenta en el híbrido de maíz INIAP H-603 con 45 número de plantas por parcela.

Tabla 6. Promedios registrados en las variables altura de planta, inserción mazorca, número de plantas por parcelas en 3 híbridos de maíz evaluados durante la época seca del año 2017 en un sistema de cultivo asociado cacao-maíz en Lodana-Manabí (INIAP Portoviejo).

\begin{tabular}{llll}
\hline Tratamientos & Altura de planta $(\mathbf{c m})$ & $\begin{array}{l}\text { Inserción de mazorca } \\
(\mathbf{c m}\end{array}$ & $\begin{array}{l}\mathbf{N}^{\circ} \text { de } \\
\text { planta/par }\end{array}$ \\
\hline $\begin{array}{l}\text { Factor A: Híbridos } \\
\text { de maíz }\end{array}$ & & & \\
INIAP H-601 & $202.21 \mathrm{a}$ & $95.92 \mathrm{a}$ & $66.71 \mathrm{a}$ \\
INIAP H-602 & $204.5 \mathrm{a}$ & $102.65 \mathrm{a}$ & $64.44 \mathrm{a}$ \\
INIAP H-603 & $168.46 \mathrm{~b}$ & $76.02 \mathrm{~b}$ & $44.90 \mathrm{~b}$
\end{tabular}

Medias con la misma letra no muestras diferencias significativas al $\mathrm{P}<0.05$ de probabilidad.

Longitud de mazorca. En el análisis de varianza para longitud de mazorca (Tabla 7), se observan diferencias altamente significativas al $\mathrm{P}<0.01$ de probabilidad entre los híbridos de maíz evaluados. La longitud de mazorca promedio fue de $18.7 \mathrm{~cm}$ y el coeficiente de variación fue de $5.54 \%$.

Diámetro de mazorca. De acuerdo al análisis de varianza para esta variable (tabla 7) se observan diferencias altamente significativas al $\mathrm{P}<0.01$ de probabilidad entre híbridos de maíz, con un coeficiente de variación de $4.59 \%$ y un diámetro de $3.5 \mathrm{~cm}$.

Porcentajes de mazorca podridas $(\%)$. Al realizar el análisis de varianza para esta variable (tabla 7) se encontraron diferencias estadísticas al $\mathrm{P}<0.05$ de probabilidad entre híbridos de maíz. Se obtuvo un coeficiente de variación de $48.33 \%$ y un promedio de mazorcas podridas de $7 \%$.

Tabla 7. Cuadrados medios para tres variables agronómicas registrados en tres híbridos de maíz, evaluados durante la época seca del año 2017. En un sistema de cultivo asociado cacao maíz en Lodana-Manabí (INIAP Portoviejo). 
Julio Gabriel, Jhon Figueroa, Ricardo Limongi, Raquel Vera, Blanca Indacochea

\begin{tabular}{|c|c|c|c|c|c|}
\hline \multirow{3}{*}{$\begin{array}{l}\text { Fuentes } \\
\text { variación }\end{array}$} & \multirow[t]{3}{*}{ de } & \multirow{3}{*}{$\begin{array}{ll}\text { Grados } & \text { de } \\
\text { libertad } & \end{array}$} & \multicolumn{3}{|c|}{ Cuadrados medios } \\
\hline & & & Mazorca & & Mazorcas \\
\hline & & & longitud (cm) & Diámetro(cm) & podridas (\%) \\
\hline Maíz & & 2 & $5.89 * *$ & $0.15 * *$ & $103.05 *$ \\
\hline Cacao & & 7 & $1.64 \mathrm{~ns}$ & $0,05 \mathrm{~ns}$ & \\
\hline Maíz*acao & & 14 & $2.36 \mathrm{~ns}$ & $0.03 \mathrm{~ns}$ & $25.46 \mathrm{~ns}$ \\
\hline Error & & 48 & $1.8 \mathrm{~ns}$ & $0.03 \mathrm{~ns}$ & $1.61 \mathrm{~ns}$ \\
\hline Total & & 71 & 1.08 & 0.03 & 2.0 \\
\hline CV $(\%)$ & & & 5.54 & 4.59 & 48.33 \\
\hline
\end{tabular}

*: Significativo al $\mathrm{P}<0.01$ de probabilidad, **: Altamente significativo al $\mathrm{P}<0.05$ de probabilidad. $\quad$ ns: no significativo.

En la tabla 8, se presentan los valores promedios y la prueba de Tukey al 5\%, aquí se observa que la variable longitud de mazorca presenta tres rangos de significancia estadística, el mayor corresponde al híbrido de maíz INIAP H-602 con $19.32 \mathrm{~cm}$ y el más bajo se presenta en el hibrido de maíz INIAP H-601 con $18.36 \mathrm{~cm}$.

Al evaluar el diámetro de mazorca entre los híbridos de maíz se determinó tres rangos de significancia estadística, el mayor corresponde al híbrido de maíz INIAP H-603 con 3.57 y el rango más bajo correspondió al híbrido de maíz INIAP H-601 con 3.41 en promedio.

Al evaluar el porcentaje de mazorcas podridas se observa que los híbridos de maíz presentan dos rangos de significancia estadística, el mayor corresponde a los híbridos de maíz INIAP H-602 e INIAP H-603 con 8.56 y $8.65 \%$ y el menor porcentajes (\%) de mazorcas podridas es el híbrido de maíz INIAP H-601 con 5.48\%.

Tabla 8. Caracteres agronómicos registrados en 3 híbridos de maíz.

\begin{tabular}{llll}
\hline Tratamientos & \multicolumn{2}{c}{ Mazorca } & Mazorcas podridas (\%) \\
& longitud $(\mathrm{cm})$ & diámetro $(\mathrm{cm})$ & \\
$\begin{array}{l}\text { Factor A: Híbridos de } \\
\text { maíz }\end{array}$ & & & \\
\hline INIAP H-601 & $18.36 \mathrm{~b}$ & $3.41 \mathrm{~b}$ & $5.48 \mathrm{~b}$ \\
INIAP H-602 & $19.32 \mathrm{a}$ & $3.50 \mathrm{ab}$ & $8.56 \mathrm{a}$ \\
INIAP H-603 & $18,63 \mathrm{ab}$ & $3,57 \mathrm{a}$ & $8.65 \mathrm{a}$ \\
Tukey P< $\mathbf{0 . 0 5}$ & 0.72 & 0.11 & 2.96
\end{tabular}

Medias con la misma letra no muestras diferencias significativas al $\mathrm{P}<0.05$ de probabilidad.

Número de hilera de grano por mazorca. En el análisis de varianza registrado para este carácter (tabla 9), se determinaron diferencias altamente significativas al $\mathrm{P}<0.01$ de probabilidad. Los híbridos evaluados registraron un promedio general de 14 hileras de granos por mazorca y un coeficiente de variación de $4.86 \%$.

Peso de 1000 granos (g). En la (tabla 9) se presenta el análisis de varianza para el peso de 1000 gramos, en el cual se detectaron diferencias estadísticas no significativas entre los híbridos

146 UNESUM-Ciencias. Publicación cuatrimestral. Vol. 6, Año 2022, No. 2 (Especial Agropecuaria) 
evaluados. El coeficiente de variación fue de $8.04 \%$ y un promedio del peso de 1000 granos de $374 \mathrm{~g}$.

Rendimiento (unidad de media). Los resultados obtenidos para esta variable se muestran en la (tabla 9), se encontraron diferencias significativas al $\mathrm{P}<0.05$ de probabilidad entre híbridos de maíz. Se obtuvo un coeficiente de variación de $32.12 \%$ y un rendimiento entre los híbridos de maíz de 1.10 t/ha.

Prolificidad. Según el análisis realizado para esta variable (tabla 9) se detectaron diferencia altamente significativa al $\mathrm{P}<0.01$ de probabilidad entre híbridos de maíz. Se registró un promedio general de 1.3 y un coeficiente de variación de $16.91 \%$.

Tabla 9. Cuadrados medios para cuatro variables agronómicas registrados en tres híbridos de maíz, evaluados durante la época seca del año 2017. En un sistema de cultivo asociado cacaomaíz en Lodana-Manabí (INIAP Portoviejo)

\begin{tabular}{|c|c|c|c|c|c|}
\hline \multirow{2}{*}{$\begin{array}{l}\text { Fuentes } \\
\text { variación }\end{array}$} & \multirow{2}{*}{$\begin{array}{l}\text { Grados } \\
\text { libertad }\end{array}$} & \multicolumn{4}{|c|}{ Cuadrados medios } \\
\hline & & $\begin{array}{l}\mathrm{N}^{\circ} \text { de hileras } \\
\text { /mazorca }\end{array}$ & Peso & Rendimiento & Prolificidad \\
\hline Maíz & 2 & $15.29 * *$ & $182.40 \mathrm{~ns}$ & $3.61 *$ & $0.42 * *$ \\
\hline Cacao & 7 & $0.66 \mathrm{~ns}$ & $153.52 \mathrm{~ns}$ & $0.11 \mathrm{~ns}$ & $0.06 \mathrm{~ns}$ \\
\hline maíz*Cacao & 14 & $0.35 \mathrm{~ns}$ & $142.13 \mathrm{~ns}$ & $0.15 \mathrm{~ns}$ & $0.04 \mathrm{~ns}$ \\
\hline Error & 48 & 0.4989 & 5.23 & $0.14 \mathrm{~ns}$ & 0.05 \\
\hline Total & 71 & 14 & 374 & 1.10 & 1.3 \\
\hline CV (\%) & & 4.86 & 8.04 & 32.12 & 16.91 \\
\hline
\end{tabular}

*: Significativo al $\mathrm{P}<0.01$ de probabilidad, **: Altamente significativo al $\mathrm{P}<0.05$ de probabilidad. $\quad$ ns: no significativo.

En la tabla 10, se presentan los valores promedios y la prueba de Tukey al $\mathrm{P}<0.05$ de probabilidad efectuada, aquí se observa que la variable número de hileras presentó tres rangos de significancia estadística, el mayor corresponde al híbrido de maíz INIAP H-603 con 15.26 el más bajo se presenta en el híbrido de maíz INIAP H-601 con 13.65.

En la variable peso de 1000 gramos (tabla 10) se observa que no hubo diferencias estadísticas significativas entre los híbridos.

Para la variable rendimiento se presentan tres rangos de significancia estadística, el de mayor rango corresponde al híbrido de maíz INIAP H-601 con 1.58 t/ha. Y el más bajo se presenta en el híbrido de maíz INIAP H-603 con 0.81 t/ha.

Al evaluar la variable de Prolificidad (tabla 10) se registraron 2 rangos de diferencias estadísticas significativa el de mayor rango corresponde al híbrido INIAP H-601 con 1.5 de Prolificidad y el más bajo se presenta en el híbrido de maíz INIAP H-603 con 1.25.

Tabla 10. Cuadrados medios para cuatro variables agronómicas registrados en tres híbridos de maíz, evaluados durante la época seca del año 2017. En un sistema de cultivo asociado cacaomaíz en Lodana-Manabí (INIAP Portoviejo). 
Julio Gabriel, Jhon Figueroa, Ricardo Limongi, Raquel Vera, Blanca Indacochea

\begin{tabular}{|c|c|c|c|c|}
\hline $\begin{array}{l}\text { Tratamientos } \\
\text { Factor A: híbridos } \\
\text { de maíz }\end{array}$ & $N^{0}$ de hileras & $\begin{array}{lll}\text { Peso de } & 1000 \\
\text { granos } & (\mathrm{g})\end{array}$ & $\begin{array}{l}\text { Rendimiento } \\
\text { (Tm/ha) }\end{array}$ & Prolificidad \\
\hline INIAP H-601 & $13.65 \mathrm{c}$ & $386 a$ & $1.59 \mathrm{a}$ & $1.51 \mathrm{a}$ \\
\hline INIAP H-602 & $14.39 \mathrm{~b}$ & $368 \mathrm{a}$ & $1.1 \mathrm{~b}$ & $1.34 \mathrm{~b}$ \\
\hline INIAP H-603 & $15.26 \mathrm{a}$ & $377 \mathrm{a}$ & $0.81 \mathrm{c}$ & $1.25 \mathrm{~b}$ \\
\hline
\end{tabular}

Medias con la misma letra no muestras diferencias significativas al $\mathrm{P}<0.05$ de probabilidad.

\section{Análisis de varianza para clones de cacao}

Altura de planta del cacao. El análisis de varianza (tabla 11), de altura de planta, indica que los coeficientes de variación (C.V. \%) se encuentran dentro de los rangos permitidos para este tipo de investigación, están entre 5.93 y $16.76 \%$. El análisis de varianza para altura de planta muestra que los cuadrados medios fueron altamente significativos al $\mathrm{P}<0.01$ de probabilidad.

Tabla 11. Cuadrados medios de la altura de planta e incremento de altura de planta en un sistema de cultivo asociado cacao-maíz en Lodana-Manabí (INIAP Portoviejo) de ocho clones de cacao.

\begin{tabular}{lllll}
\hline F.V. & gl & Altura Eva 1 & Altura Eva 2 & Incremento Alt \\
\hline Modelo & 9 & 52.84 & 62.37 & 5.52 \\
Trat & 7 & $755.9 * *$ & $67.54 * *$ & $6.58 \mathrm{~ns}$ \\
Rep & 2 & $42.13^{* *}$ & $44.27 * *$ & $1.84 \mathrm{~ns}$ \\
Error & 14 & 7.7 & 11.18 & 9.52 \\
Total & 23 & & & \\
CV(\%) & & 5.93 & 5.12 & 16.76
\end{tabular}

**,*: Significativo al 1 y $5 \%$ de probabilidad, respectivamente ns: Diferencias estadísticas no significativas.

Para la variable de altura de planta en el cultivo de cacao se encontraron diferencias entre tratamientos, destacando tanto en la evaluación 1 y 2 el clon EET-800 con valores de 55.3 y $75.63 \mathrm{~cm}$ respectivamente. Sin embargo, los 7 tratamientos restantes fueron estadísticamente iguales. Por lo tanto, en el incremento se detectó diferencias

Diámetro de planta del cacao. Al realizar el análisis de varianza para esta variable (tabla 12) en la evaluación 1, se detectaron diferencias altamente significativas al $\mathrm{P}<0.01$ de probabilidad entre clones de cacao. Esto indica que al menos un tratamiento es diferente. Se observa un coeficiente de variación de $5.08 \%$ y un diámetro de tallo entre todos los clones evaluados $0.7 \mathrm{~cm}$.

Tabla 12. Cuadrados medios de diámetro de tallo evaluado en ocho clones de cacao asociado cacao-maíz en Lodana-Manabí (INIAP Portoviejo) de ocho clones de cacao.

\begin{tabular}{lllll}
\hline F.V. & Gl & Diámetro Eva1 & Diámetro eva2 & Incremento Diat \\
\hline Modelo & 9 & 0.01 & 0.01 & 0 \\
Trat & 7 & $0.01^{* *}$ & $0.01 \mathrm{~ns}$ & $0 \mathrm{~ns}$ \\
Rep & 2 & $0.01^{* *}$ & $0.01 \mathrm{~ns}$ & $0 \mathrm{~ns}$ \\
Error & 14 & 0 & 0.01 & 0
\end{tabular}

148 UNESUM-Ciencias. Publicación cuatrimestral. Vol. 6, Año 2022, No. 2 (Especial Agropecuaria) 
Publicación cuatrimestral. Vol. 6, No. 2 (Especial Agropecuaria), Año 2022. Pág. 135-152 COMPORTAMIENTO DE HIBRIDOS DE MAIZ (Zea Mays L.) EN SISTEMA TRANSITORIO CON CACAO

Total 23

$\operatorname{CV}(\%)$ 5.08 7.21

19.38

**,*: Significativo al 1 y $5 \%$ de probabilidad, respectivamente ns: Diferencias estadísticas no significativas.

Para la variable diámetro de tallo para el cultivo de cacao, en la evaluación 1, se encontró diferencias entre tratamientos, encontrándose dos rangos de significancia estadística destacando el clon EET-800 con valores de $0.77 \mathrm{~cm}$ ocupando el primer rango, mientras el rango más bajo lo presentó el clon EET-576 con valores de $0.63 \mathrm{~cm}$.

Para la evaluación 2 y para el incremento en el diámetro de tallo no se presentaron diferencias estadísticas significativas.

La comparación de medias de la primera evaluación de cobertura de cacao realizada (tabla 13), detecto diferencias altamente significativas al $\mathrm{P}<0.01$ de probabilidad entre clones de cacao. Esto indica que al menos un tratamiento es diferente. Se observó un coeficiente de variación de 3.53\% y una cobertura de cacao con promedio de $28 \%$, entre los ocho clones. Sin embargo, tanto para la evaluación dos e incremento de cobertura vegetal no se detectaron diferencias estadísticas significativas.

Tabla 13. Cuadrados medios de cobertura vegetal evaluada en ocho clones de cacao asociado cacao-maíz en Lodana-Manabí (INIAP Portoviejo).

\begin{tabular}{lllll}
\hline F.V. & gl & Cob Eva 1 & Cob Eva 2 & Incremento Cob \\
\hline Modelo & 9 & 958 & 58.77 & 44.76 \\
Trat & 7 & $74.36 * *$ & $57.65 \mathrm{~ns}$ & $40.42 \mathrm{~ns}$ \\
Rep & 2 & $0.73 \mathrm{~ns}$ & $62.66 \mathrm{~ns}$ & $59.91 \mathrm{~ns}$ \\
Error & 14 & 0.98 & 42.88 & 42.07 \\
Total & 23 & & & \\
CV(\%) & & 3.53 & 11,81 & 23.63 \\
\hline
\end{tabular}

Para la variable cobertura vegetal en el cultivo de cacao de acuerdo al análisis de varianza se encontraron diferencias significativas entre los tratamientos de la evaluación 1, agrupando cuatro categorías estadísticas destacando el clon EET801con 36.51\% ocupando el primer rango de significancia estadísticas de cobertura vegetal, seguido por los clones EET 454 y EET 103 con valores de 31.7 y 31.1 respectivamente.

\section{Discusión}

Limongi et al. (2011) en un estudio realizado sobre el comportamiento del maíz en un sistema agroforestal, con árboles dispersos en la cuenca del Río Carrizal, determinaron que los árboles dispersos ofrecen limitantes para la producción de maíz porque compiten con este por agua y luz. En nuestra investigación no observamos este efecto, debido a que las plantas de cacao estaban aún pequeñas, lo cual nos permitió evidenciar que los híbridos de maíz mostraron buen comportamiento, determinándose un alto rendimiento del maíz, mayor prolificidad, mayor longitud de mazorca y menor porcentaje de mazorcas podridas en el hibrido INIAP H-601; 
Julio Gabriel, Jhon Figueroa, Ricardo Limongi, Raquel Vera, Blanca Indacochea

aunque en experimentos previos esté hibrido no se comportó bien en monocultivo (INIAP, 2017). Asimismo, se observó que el híbrido mencionado mostro una mayor altura respecto a los demás híbridos evaluados. Se debe resaltar que Berrocal (2012) contrariamente a los resultados que hemos encontrado, no observó ningún efecto del maíz en el número de mazorcas en un sistema de café.

Cabe mencionar que los productores conocen cuáles especies son más compatibles con el maíz y cuáles son menos compatibles. Según Limongi et al. (2003), las especies de mayor preferencia son Prosopis spp, seguida de Cordia alliodora y Albizia guachapele debido a que necesitan de poco manejo, dan poca competencia al maíz, dan leña y forraje y generan ingresos económicos por la venta de madera, horcón y carbón. Este aspecto no fue evidenciado en nuestra investigación, pero es válido desde el punto de vista del manejo de las especies en el sistema cacao-maíz.

Fue notorio observar en nuestro experimento que el maíz aparentemente tuvo una influencia positiva sobre el crecimiento del cacao EET-800.

Aun cuando se observaron diferencias en la floración masculino, femenino y el acame, no podemos aseverar si esto fue debido a la interacción con el cacao. Lo que si observamos fue que los maíces híbridos tuvieron diferente comportamiento.

Un aspecto que cabe resaltar es que en las parcelas de investigación no observamos ataques de Spodoptera frugiperda, lo cual está indicando que posiblemente el sistema cacao-maíz, contribuye al control cruzado de este tipo de plagas.

\section{CONCLUSIONES}

La investigación permitió determinar los efectos positivos del sistema de asociación maíz cacao, evidenciando que los híbridos de maíz mostraron buen comportamiento, alto rendimiento, mayor prolificidad, mayor longitud de mazorca y menor porcentaje de mazorcas podridas en el hibrido INIAP H-601.

El híbrido de mayor rendimiento de maíz (Zea mays) asociado al cultivo de cacao (Theobroma cacao) fue el INIAP-H-601 con 1.59 t/a, y una Prolificidad de 1.51 mazorcas por planta en promedio.

Se observó que el maíz aparentemente tuvo una influencia positiva sobre el crecimiento y el incremento del diámetro del cacao EET-800, esto se denotó asimismo la no presencia de plagas importantes del maíz, como Spodoptera frugiperda.

Fue notorio la variabilidad de las plantas de cacao frente a la asociación con maíz.

La influencia del maíz en la dinámica del crecimiento del cacao es positiva, presentando un incremento de altura de planta de $37.37 \mathrm{~cm}$ en el clon de cacao EET-800 y un incremento de diámetro de $0.77 \mathrm{~cm}$ esto denota que el clon EET-800 es el que mejor se adapta a este sistema cacao - maíz.

\section{REFERENCIAS BIBLIOGRÁFICAS}

150 UNESUM-Ciencias. Publicación cuatrimestral. Vol. 6, Año 2022, No. 2 (Especial Agropecuaria) 
Alviar, R. E. (2016). Sistema de Producción Agroforestal. Manual de Modelos Productivos con Efectividad Climática, 5-6. https://www.tncmx.org/content/dam/tnc/nature/en/documents/mexico/M-REDDManualModelosProductivosEfectividadClimatica_2016.pdf

ANECACAO. (2017). Asociación Nacional de Exportadores de Cacao - Ecuador. http://www.anecacao.com/es/noticias/industria-estadounidense-dechocolates-tendencias-estimaciones-yoportunidades-al-2018.html

Berrocal, A. M. (2012). Productividad de café en Sistemas Intercalados. Colombia. https://www.cenicafe.org/es/documents/LibroSistemasProduccionCapitulo11.pdf

CIMMYT, 1. (1995). Manejo de los ensayos informe de los datos para el Programa de Ensayos Internacionales de Maíz del CIMMYT. México, D.F. (Quinta reimpresión, 1999. ed.). México. https://repository.cimmyt.org/xmlui/bitstream/handle/10883/764/68309.pdf?sequen ce=1\&isAllowed=y

Enríquez, G. (2010). Cacao orgánico: Guía para productores ecuatorianos (2a ed.). *EC-INIAP-BEETP-MBY. Quevedo (INIAP/M-54). Quito, Ecuador. http://repositorio.iniap.gob.ec/handle/41000/4571

Figueroa, J. y Gabriel J. (2019). Comportamiento de híbridos de maíz (Zea mays L.) como sistema asociado en el cultivo de cacao (Theobroma cacao L.) en la Parroquia Lodana. Tesis, Universidad Estatal del Sur de Manabí, Jipijapa, Manabí, Ecuador. http://repositorio.unesum.edu.ec/bitstream/53000/1813/1/UNESUM-ECUING.AGROPE-2019-05.pdf

Iniap. (2017). Productos elaborados y semielaborados. http://www.manabi.gob.ec/investmanabi/Exportaciones.php

IPGRI (1991) Instituto Internacional de Recursos Fitogenéticos.Instituto de Investigación Internacional con el mandato de avanzar en la conservación y el uso de la Diversidad Genética para el bienestar de las generaciones actuales y futuras. Centro del Grupo Consultivo sobre Investigación Agrícola Internacional (CGIAR). http://www.bioversityinternational.org/Plants_and_Animals/Forests_and_Trees/index.asp

Gabriel, J., Castro, C., Valverde A \& Indacochea B (2017). Diseño Experimentales. Grupo Compas, Universidad Estatal del Sur de Manabí, Jipijapa, Ecuador. http://repositorio.unesum.edu.ec/handle/53000/2064

Limongi, R. (2011). Caracterización y diversidad florística del sistema agroforestal maíz con árboles dispersos en la cuenca del Carrizal, Manabí Ecuador. Plan de Fortalecimiento Institucional. http://www.iniap.gob.ec/nsite/images/documentos/caracterizacion-y-diversidadfloristica-del-sistema-agroforestal$1 . p d f$

Melo C, Hollander G. (2013). Unsustainable development: Alternative food networks and the Ecuadorian Federation of Cacao Producers, 1995-2010. Journal of Rural Studies 32:251-263.

Quiroz, J., \& Mestanza, S. (2012). Establecimiento y manejo de plantaciones de cacao. Obtenido de Estación Experimental Litoral del Sur. Programa Nacional del Cacao. http://infocafes.com/portal/wpcontent/uploads/2016/12/boletin_146_establecimiento_y_manejo_de_una_plantaci $\%$ C3\%B3n_de_cacao.pdf

Rojas, J. G. (2015). Manual Técnico del Cultivo de Maíz Bajo Buenas. Gobernación de Antioquia, Secretaría de Agricultura y Desarrollo. Medellín, Colombia. https://es.scribd.com/document/357086162/MANUAL-DELCULTIVO-DE-MAIZ-pdf

Salazar David; Villafuerte Walter; Cuichán Maritza; Orbe Diego \& Márquez Julio (2016). Encuesta de Superficie y Producción Agropecuaria $\quad$ Continua 2016. http://www.ecuadorencifras.gob.ec/documentos/webinec/Estadisticas_agropecuarias/espac/espac2016/Informe\%20ej ecutivo\%20ESPAC_2016.pdf

SINAGAP. (2016). Rendimientos de maíz duro seco en invierno. http://sinagap.agricultura.gob.ec/pdf/estudios_agroeconomicos/rendimiento_maiz_d uro_seco_invierno2016.pdf

Somarriba, E y Beer, S. (1994). Maderables como alternativa para la sustitución de sombras en cacaotales establecidos: el concepto CATIE.Serie Técnica 238, 34. 
Julio Gabriel, Jhon Figueroa, Ricardo Limongi, Raquel Vera, Blanca Indacochea

152 UNESUM-Ciencias. Publicación cuatrimestral. Vol. 6, Año 2022, No. 2 (Especial Agropecuaria) 